\title{
Automotive user interfaces and interactive applications in the car
}

\author{
Andrew L. Kun • Albrecht Schmidt • \\ Anind Dey $\cdot$ Susanne Boll
}

Published online: 7 April 2012

(C) Springer-Verlag London Limited 2012

In-vehicle electronic devices are becoming ubiquitous. Drivers and passengers use these devices because they perceive them as providing valuable services. Some of these devices, such as collision warning systems, assist drivers in performing the primary task in a vehicle that is driving; others provide information on myriad subjects or entertain the driver and passengers. A problem that arises from the proliferation of in-vehicle devices is that they might distract drivers from the primary task of driving, with possibly disastrous results.

Thus, it is no surprise that a large number of research and development efforts in industry and academia explore the user interfaces of in-vehicle electronic devices, with the ultimate goal of making interactions with the devices safe as well as useful and enjoyable. These efforts include designing interfaces using various interaction modalities (such as

\author{
A. L. Kun $(\bowtie)$ \\ Electrical and Computer Engineering Department, \\ University of New Hampshire, Kingsbury Hall, \\ Durham, NH 03824, USA \\ e-mail: andrew.kun@unh.edu

\section{A. Schmidt \\ Computer Science Department, Institute for Visualization \\ and Interactive Systems, University of Stuttgart, \\ 70569 Stuttgart, Germany \\ e-mail: Albrecht.Schmidt@vis.uni-stuttgart.de}

\author{
A. Dey \\ HCI Institute, Carnegie Mellon University, \\ NSH 2502C, 5000 Forbes Ave., Pittsburgh, \\ PA 15213-3891, USA \\ e-mail: anind@cs.cmu.edu \\ S. Boll \\ Department of Computing Science, University of Oldenburg, \\ 26129 Oldenburg, Germany \\ e-mail: susanne.boll@informatik.uni-oldenburg.de
}

speech, vision, and touch), assessing the impact of interactions on the driver (e.g., using measures of cognitive load and visual demand), and creating benchmarks for assessing the impact of in-vehicle interactions on drivers (such as the lane change task and the peripheral detection task). The efforts often focus on specific in-vehicle tasks, such as navigation or music selection, because user interface designers have an immediate need for application-specific guidelines.

This theme issue is part of the guest editors' effort to create a vibrant community of researchers and developers involved in exploring user interfaces in vehicles. The focal point of our effort is the Automotive User Interfaces and Interactive Vehicular Applications (AutomotiveUI) conference, which is now in its fourth year. Many of the 17 papers submitted in response to our call for this theme issue came from the participants of prior AutomotiveUI conferences. In a highly selective review process, based on reviews provided by approximately 50 experts in the field, three of these submissions were selected for publication. One of these papers focuses on improving human-computer interaction for a specific application (navigation), while the other two explore the use of two different interaction modalities (manual-visual and speech) in vehicles.

\section{Improving navigation systems}

In their paper entitled "On Credibility Improvements for Automotive Navigation Systems," Schaub, Hipp, Kargl, and Weber argue that credibility, "a perceived quality that reflects the trustworthiness and expertise of a system," is an often-neglected aspect of in-vehicle user interfaces. However, designers neglect this issue at their own peril, as users will likely dismiss a system with low credibility, and this can negatively affect the reputation of the product and 
the associated brand. Schaub and colleagues identify a number of interaction weaknesses that harm the credibility of today's navigation systems and propose mechanisms to overcome these weaknesses.

\section{Exploring the use of two interaction modalities}

In "Browsing the Information Highway while DrivingThree In-Vehicle Touch Screen Scrolling Methods and Driver Distraction," Kujala finds that using kinetic scrolling, a popular scrolling method implemented on many smartphones, is associated with high visual demand. Thus, Kujala recommends avoiding the use of kinetic scrolling by drivers and suggests that page-by-page touch swiping might be appropriate for use while driving.

Kun, Shyrokov, and Heeman are interested in building in-vehicle speech user interfaces that allow interaction with multiple devices to overlap in time. In "Interactions between Human-Human Multi-Threaded Dialogues and Driving," they suggest that interface design can be inspired by dialogue behaviors observed in human spoken interactions. They elicit a number of such behaviors in a driving simulator experiment and report that switching between verbal tasks can increase the driver's cognitive load. However, they also present evidence that some dialogue behaviors might actually reduce the driver's cognitive load.

\section{Conclusions}

The three papers in this theme issue are a sample of the exciting research by the members of our fledgling community. They portray this community as being multi-disciplinary, with roots in the fields of human-computer interaction and ubiquitous computing. They also highlight the fact that our community is very much focused on designing safe in-vehicle user interfaces, and that much of our work is aimed at designing specific applications such as navigation and music selection. Finally, the papers underscore the fact that members of our community are not bound by convention (in contrast to many research and development efforts in car companies) and that they push the boundaries of in-vehicle interface design. The guest editors would like to encourage readers who are intrigued by these papers to consider engaging our community, for example by participating in one of the upcoming AutomotiveUI conferences (http://www.auto-ui.org).

Acknowledgments We would like to thank PUC Editor-in-Chief Peter Thomas for his support throughout the publication process of this theme issue. We would also like to thank the reviewers for contributing their time and expertise to select the best papers for this theme issue and for helping to improve the papers selected for publication. Finally, we would like to thank all the authors who submitted their work to this theme issue. 